\title{
Influence of mechanical ventilation and cooling systems on vibrations of high precision machines
}

\author{
Lukasz Scislo, ${ }^{1, *}$, and Nina Szczepanik-Scislo ${ }^{2}$ \\ ${ }^{1}$ Faculty of Electrical and Computer Engineering, Tadeusz Kosciuszko Cracow University of \\ Technology, ul. Warszawska 24, 31-155 Krakow, Poland \\ ${ }^{2}$ Faculty of Environmental Engineering, Tadeusz Kosciuszko Cracow University of Technology, \\ ul. Warszawska 24, 31-155 Krakow, Poland
}

\begin{abstract}
The aim of the research was to describe the effects that air and water cooling systems can have on the dynamic behaviour of precise machines. Although much thought is paid to vibration isolation of the ground and isolation of individual effects of machines operating close to each other, it is often forgotten to model or to measure the effects that the ventilation or the machines cooling systems have on the machine itself. This can be especially important for high precision machines used for experimental research and medical equipment. The article shows the effects of ventilation and cooling system on the induction of additional resonant frequencies of the system for the high precision machine like a linear collider. This kind of machine requires special environmental conditions to assure proper beam stability. Due to the dynamic behaviour of typical machines, the presence of the new high amplitude frequencies in the $0-100 \mathrm{~Hz}$ range is very dangerous for its stability of work. In the case of high precision machines, it is not only a cause of not optimal working conditions but very often is a cause of serious problems.
\end{abstract}

\section{Introduction}

One of the visible effects of the dynamical behaviour of any kind of working machinery are the vibrations induced in the system. The increase of the vibration levels may be caused by misalignment of parts, not enough structure stiffness or wrong connections. The dynamic conditions may also change due to the changes in environmental conditions like air pressure, temperature, humidity, ventilation conditions or a combination of these parameters. This is why the dynamic behaviour monitoring of machines (measurements of the amplitude of vibration at certain frequencies) is especially beneficiary to provide definite information about the accuracy, stability of working conditions and possible critical problems with certain parts or the whole system. Vibration monitoring is also a way to examine the effect and possibility of resonance occurrence. This effect is often present when a machine part (e.g. casing, piping and supply lines, supporting structures) or a machine working in the

* Corresponding author: 1 scislo@.pk.edu.pl 
vicinity have similar dynamical behaviour at certain frequencies (resonant frequencies). Therefore, dynamic behaviour measurements and analysis are an effective, non-intrusive method to monitor machine condition during initialisations, planned or unplanned terminations and during normal operations [2], especially when using non-contact measurement methods [1]. This is also the way to perform determinations of the operating and mechanical condition of equipment, which further can be used for system evaluation [3]. In consequence, the complex vibration monitoring of past, current and estimation of future behaviour can be the ideal way to identify developing problems before they become too serious and cause unscheduled termination of machines operations [4]. The unexpected shutdown and additional repairs, services and calibrations can be avoided by scheduling regular monitoring of machine vibrations either on the basis of chosen intervals or in a continuous manner [5-6]. Consequently, increasing amplitudes of vibration levels can identify flawed maintenance practices including mistakes in the installation process, different material used for replacements, parts misalignments or wrong isolation from ground motion conditions [7-8]. It can also allow the detection of new environmental influence from nearby operating devices and systems or e.g. civil engineering operations [4, 9].

\section{Methods}

\subsection{The test object}

To evaluate vibration influence on the high precision machines a test object very sensitive to vibration was chosen. It was decided to perform the measurements in the test chamber of Compact Linear Collider (CLIC), which could be a device to open the way to new physics and additionally to proof or disproof current theories [10]. The collider is a two-beam acceleration design, which imposes restrictive requirements on its components to assure the beam stability. One of the most important technical challenges, this accelerator needs unprecedented ground motion suppression techniques. This is especially important having in mind that the nominal centre of mass energy of $3 \mathrm{TeV}$ and generation a high accelerating gradient of $100 \mathrm{MV} / \mathrm{m}[10,11]$. One of the sources of dynamic misalignments are the ground motion conditions and their effect on supporting structure and the accelerator itself $[12,13]$. The second source often comes from dynamical behaviour of the environment and additional equipment and system operating in the close distance.

\subsection{Measurement equipment and setup}

To perform vibration monitoring, especially to record ground motion, proper instrumentation was needed. This consisted of a dedicated sensor and an acquisition system with signal processor $[14,15]$. When considering the ground motion the most common equipment is a geophone. A geophone consists of three independent sensing units, one in the vertical direction $(Z)$, and the other two in horizontal directions (X and Y respectively) [11]. In case of dynamic behaviour examination of the specific equipment or structure, smaller sensors called accelerometers are often used. For this study two highly sensitive geophone sensors (G1, G2) shown in Fig.1, were used in order to perform ground vibration measurements in the test area. Additionally, three precise seismic accelerometers PCB 393B31 (S1, S2, S3) were used to measure the modules acceleration. To measure the dynamic response of the structure, two tri-axial accelerometers PBC 356B08 (A4, A5) were also placed on the structures girder and the magnet itself. General (ambient) vibration measurements were performed using 2 high precision geophones placed on the ground. They were located inside 
the laboratory chamber with the examined machine, while three of the seismic accelerometers were fixed on top of the module in vertical ( 2 seismic accelerometers, one on the structure and one on the girder) and horizontal direction (1 seismic accelerometer on the girder). Fig. 1 shows the placement of sensors within the chamber.
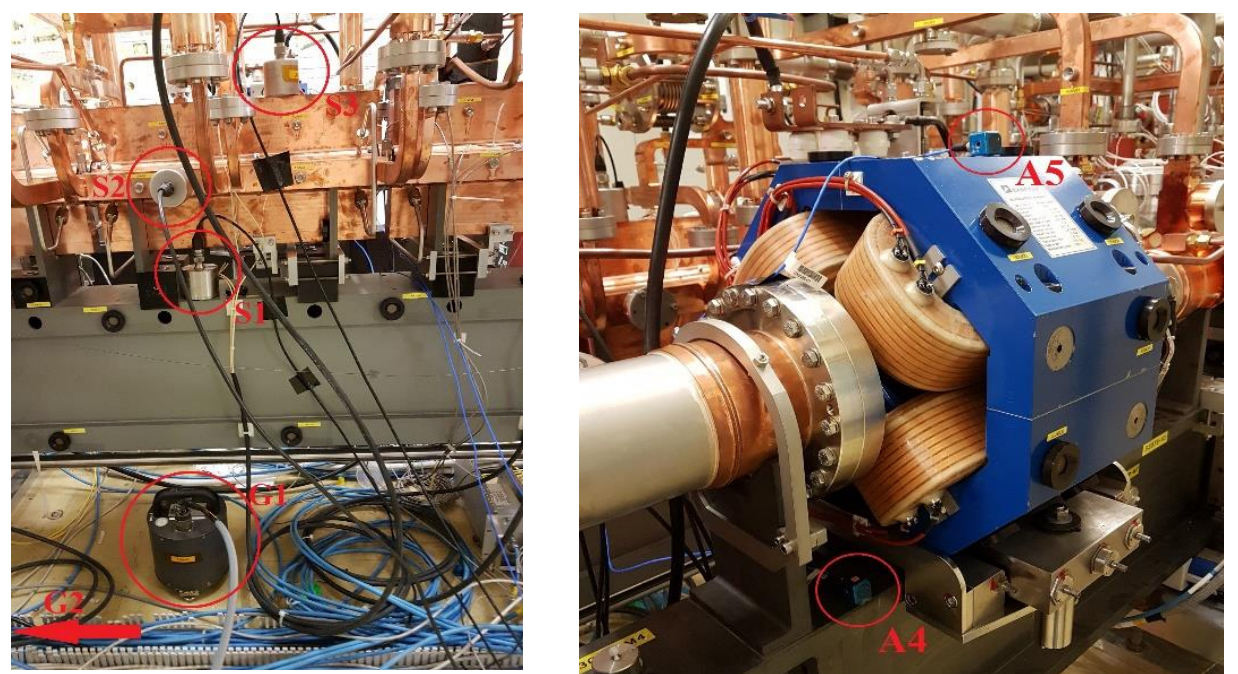

Fig. 1. Placement of the sensors on the ground, girder and structure (left) and on the magnet (right).

The measurement followed the scheme shown in Table 1. During a specific time of the day, different subsystems (air, water cooling systems) would be turned on or off, thus allowing to determine the difference in vibration levels, its contribution and excitation frequencies or each of the subsystems.

Table 1. Measurements scheme.

\begin{tabular}{|c|c|c|c|c|}
\hline $\begin{array}{c}\text { Mes. } \\
\text { No. }\end{array}$ & Air cooling & $\begin{array}{c}\text { Flow } \\
{[\% \text { of max.] }}\end{array}$ & Water cooling & $\begin{array}{c}\text { Flow } \\
{[\% \text { of } \max ]}\end{array}$ \\
\hline 1 & OFF & 0 & OFF & 0 \\
\hline 2 & ON & 50 & OFF & 0 \\
\hline 3 & OFF & 0 & ON & 50 \\
\hline 4 & OFF & 0 & ON & 100 \\
\hline 5 & ON & 100 & ON & 100 \\
\hline 6 & ON & 100 & OFF & 0 \\
\hline
\end{tabular}

The principle of work of the air and water cooling system is shown in Fig 2.


Fig. 2. An air cooling [16] and a water cooling system overview [17]. 
The maximal airflow was set to $0.8 \mathrm{~m} / \mathrm{s}(100 \%)$. An additional measurement was taken with $50 \%$ of maximal airflow $(0.4 \mathrm{~m} / \mathrm{s})$. In principle, the water cooling system should not cause problems in the measured frequency range (due to the high stiffness of the structure. However, additional measurements were taken to prove this assumption.

\section{Discussion of results}

When considering systems with dynamic conditions like in the study, it is beneficiary to use a sweep excitation in the frequency range. This is a useful indicator which allows a good overview of current dynamic conditions. It can also provide information about the effectiveness of vibration isolation [18]. This approach to the system diagnostics requires a detailed and accurate frequency analysis. It is especially dangerous for the given system if distortion of harmonic frequencies happens. This often results in matching this frequency value with one of the resonant ones and can result in magnification of amplitudes. If it occurs it may force the designer to change the responsible element or redesign the whole system. Moreover, ground vibration from sources such as working civil engineering machinery, landing planes or road traffic can be a big influence on the vibratory conditions measured and in some cases can be observed by the building occupants [4].

\subsection{Ambient vibration measurement}

Recording ambient vibrations of the examined area required an acquisition system, which records data in a time domain and later processed that date in the frequency domain. These dynamic conditions are the effect of different forces coming from working ventilation system, wind, traffic noise, some excitation coming from $\mathrm{p}$ and $\mathrm{s}$-waves from earthquakes and other small changes in the environment. This why the ambient vibration measurement technique is beneficiary both to describe dynamical conditions of the environment but can also be used as a structural health monitoring [19].

These background vibration measurements were done in order to study the effects of the normal working conditions in the building and traffic effect from the nearby road. The measurements were done about 15 meters from public roads. Additionally, a small machining workshop was located in the neighbouring room, which also can be a source of additional vibrations.
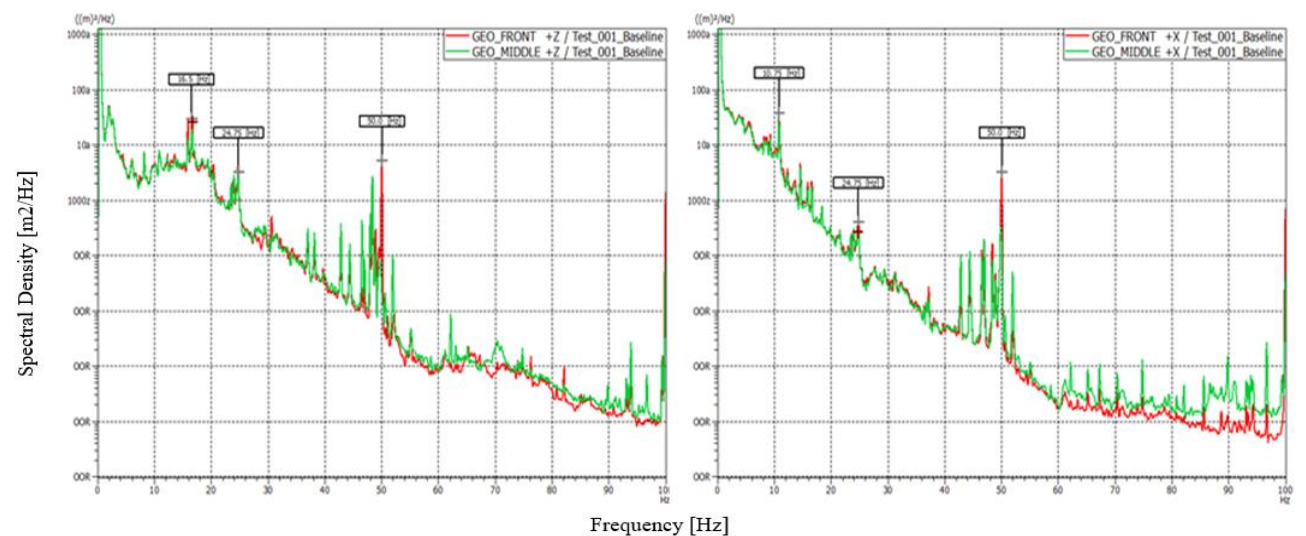

Fig. 3. Power Spectral Density for vertical (left) and horizontal (right) ground vibration in the examined room. 
The measurement set up allowed the observation of dynamic conditions in the examined room. Furthermore, this was a start point to evaluate how ground vibration is transferred to objects, including the examined machine in the test area.

\subsection{Integrated RMS vibration comparison between the baseline and situation of all system in operation}

The integrated RMS (Root Mean Square) value was used to add up the total vibration in a spectrum, including ambient. The measurement data was taken in 20 minutes time blocks during the normal working day. During post-processing, the power spectral density was calculated. Integrated displacement from $1 \mathrm{~Hz}$ to $100 \mathrm{~Hz}$ in the vertical direction was calculated by the sensor (geophone) located on the ground (Fig. 4 left) and a sensor located on the examined structure (Fig. 5 right). Each time conditions were examined for the case without the presence of ventilation and water cooling system (ALL_OFF) and both systems working with maximal power (ALL_ON).
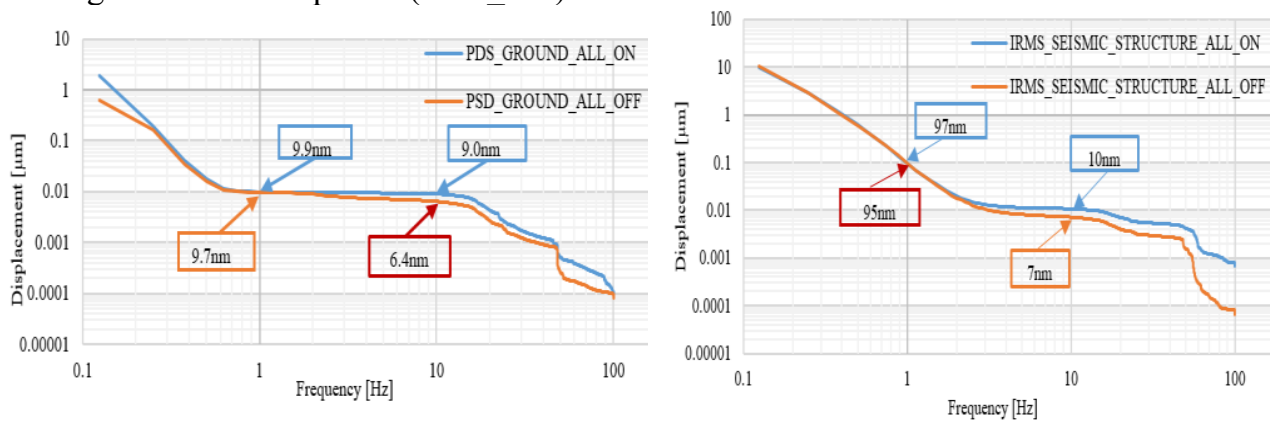

Fig. 4. Integrated RMS vibration comparison (vertical) between the baseline and situation of all system on (air cooling 100\%, water cooling 100\%). Geophone (G2) located on the ground (left) and a seismic sensor located on the structure (right).

Additionally, these values were compared with additional locations (Table 2) associated with low vibration conditions both on the surface and underground.

Table 2. IRMS results measured in different locations.

\begin{tabular}{|c|c|c|}
\hline Measurement place & IRMS at 1 Hz [nm] & IRMS at 10 Hz [nm] \\
\hline Examined room & 9.7 & 6.4 \\
\hline $\begin{array}{c}\text { Mechanical Measurement } \\
\text { Laboratory (CERN) }\end{array}$ & 17.4 & 16.7 \\
\hline $\begin{array}{c}\text { Metrology Laboratory } \\
\text { (CERN) }\end{array}$ & 11.7 & 10.1 \\
\hline LHC tunnel (CERN) & 1.9 & 0.5 \\
\hline
\end{tabular}

As it is visible, the room had very good dynamic conditions in comparison with other surface placements. LHC tunnel, located $100 \mathrm{~m}$ below the surface is included here as a reference and has 10 times lower level of the typical building structures at the surface. This was one of the main reasons to place the experiments underground and assure the stability of the LHC work. 


\subsection{Ventilation and cooling system influence on the vibration levels (separate system in operation)}

The following graphs show the results for vertical $(Z)$ and lateral $(X)$ direction. The measurements were made using uniaxial seismic accelerometers. Due to their high accuracy, they present reliable measurement results for changing conditions within the system. The baseline measurement was conducted without any system working with normal activity in the building and nearby road. The data was collected over a period of over 20 minutes.

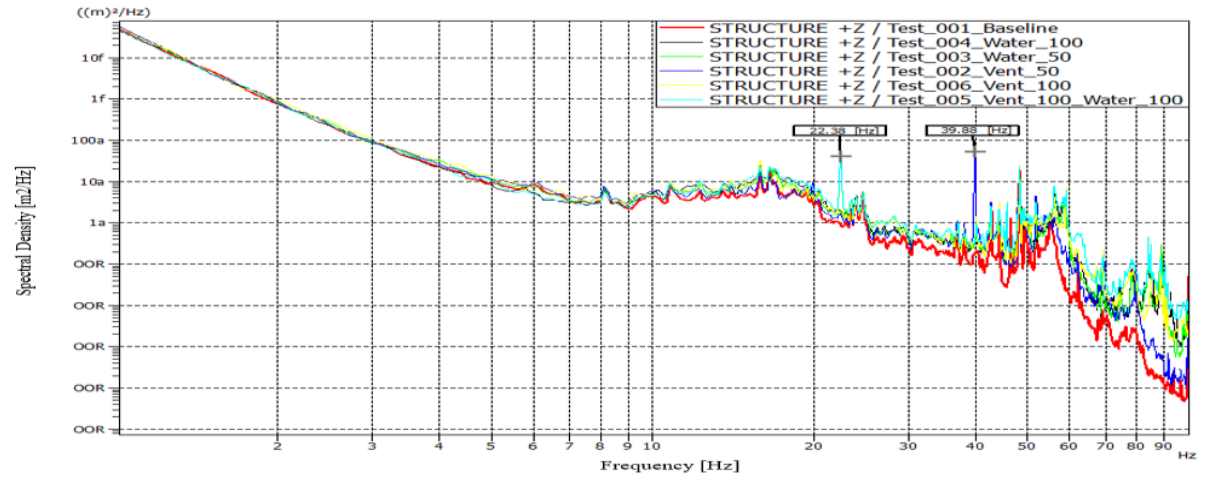

Fig. 5. Power Spectral Density for vertical (V) vibration of the module.

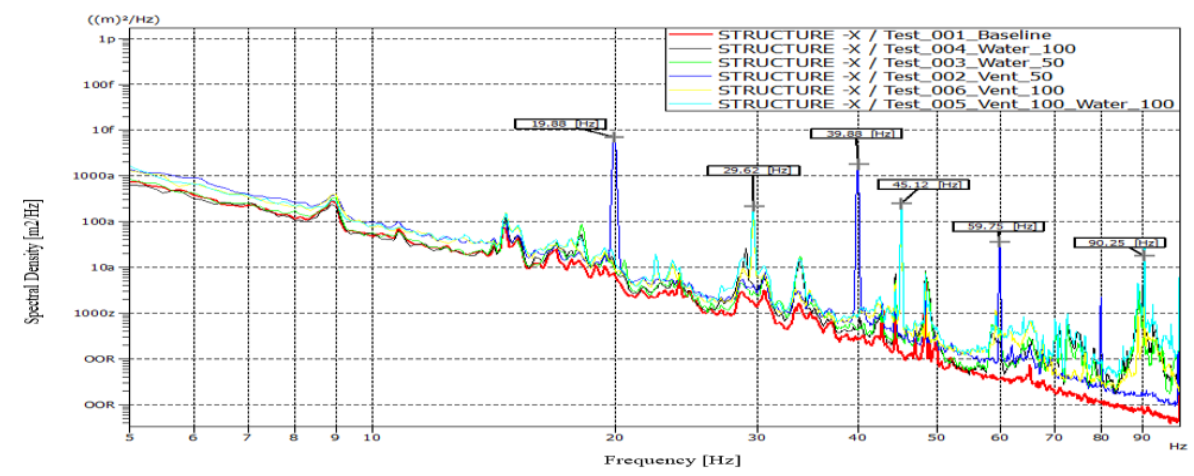

Fig. 6. Power Spectral Density for lateral (EW) vibration of the module.

As expected, new frequencies were observed due to the excitation from the ventilation and water cooling system (Fig. 5 and 6). The new picks were present with both settings of the airflow of the ventilation system $(50 \%, 100 \%)$. Additional frequencies were observed while both systems were in operation (ventilation and water cooling). Specifically, the ventilation systems working at $50 \%$ of the power excites frequencies $19.88 \mathrm{~Hz}, 39.88 \mathrm{~Hz}$, $59.75 \mathrm{~Hz}$ (Fig.6). Moreover, the ventilation system working on $100 \%$ of the power shows the excitation of additional frequencies at $29.62 \mathrm{~Hz}$ and $45.12 \mathrm{~Hz}$ (Fig.6).

Specific frequencies that were excited are clearly visible. Specifically, $22.38 \mathrm{~Hz}, 48.38$ $\mathrm{Hz}, 56.25 \mathrm{~Hz}, 84 \mathrm{~Hz}$ (vertical direction) are the frequencies excited by the air and water cooling systems. Additional vibration may cause the misalignment of the beam and in consequences failure to produce the data for the experiments or even danger to the machine and operators. 


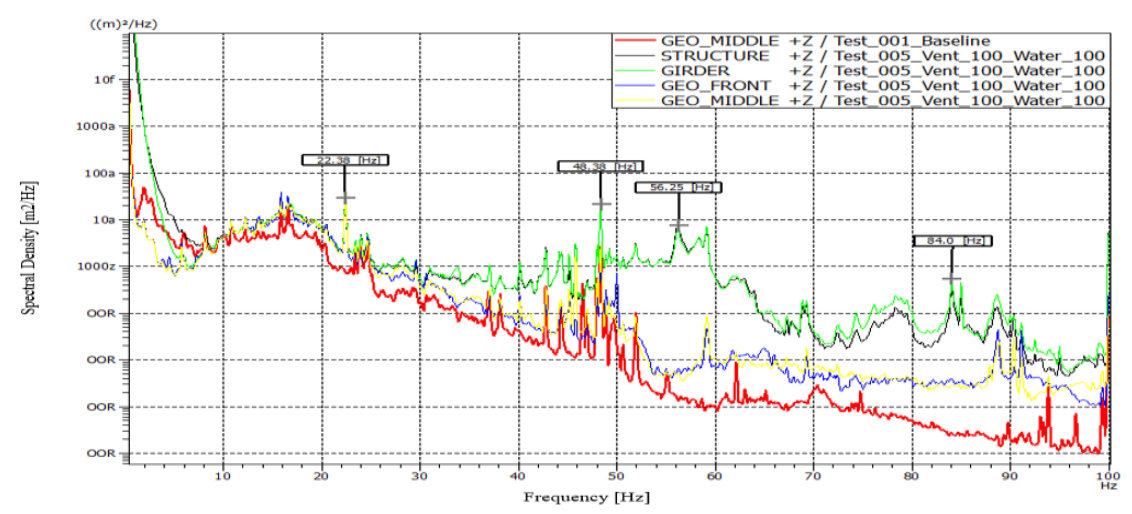

Fig. 7. Power Spectral Density for vertical (V) vibration of the module.

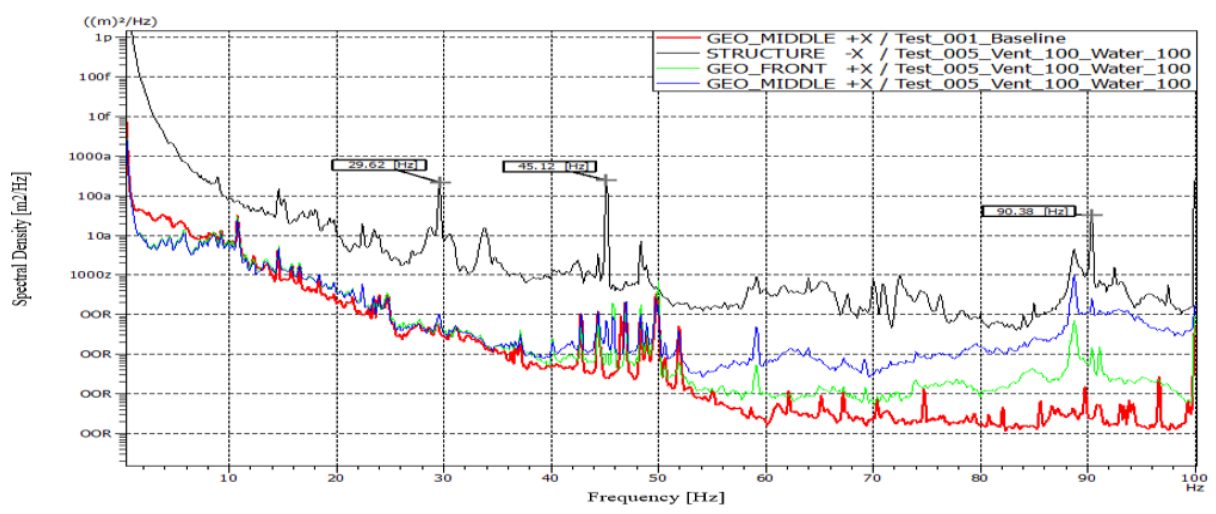

Fig. 8. Power Spectral Density for lateral (EW) vibration of the module.

\section{Conclusion and observations}

The goal of the measurements was to measure the levels of vibration caused to the module by its subsystems (air cooling, water cooling). An associated goal was to compare the level of vibration with all systems operating at full capacity. This allowed to observed the effect that those systems have on the experiments. The vibration of high precision equipment and research aperture is influenced by the operation of nearby machinery. This is visible in the PSD graphs corresponding to the ground vibration measured by the geophones located next to the examined module (Fig. 3). Additionally, there was an increase in the integrated level of vibration (compared at $1 \mathrm{~Hz}$ ) between all systems disabled and all systems in operation (Fig. 4). This increase in vibration levels between the ground and the structure was expected but its level clearly proves the big influence of the cooling and ventilation systems on the modules dynamics. The comparison of PSD graphs for the situation with all subsystem turned off and all subsystems turned on (Fig. 7 and 8), show the expected increase of vibration levels in the latter case. Several peaks are visible in the graphs for the module corresponding to the natural frequencies of the structure and the girder. This situation not only had an influence on the results of the experiments, but also on the safety and stable operations of the whole structure. Prolonged exposure to this additional excitation will result also in fatigue problems. Therefore, the additional vibration will affect the alignment of the parts and cause the necessity to make corrections and repairs. Additionally, when evaluating both systems separately, the influence of the air cooling system on vibrations levels was greater than the influence of the water cooling system. However, the work of both systems simultaneously 
effects in additional excitations (Fig. 5). This allowed also to invalidate the assumption that due to its high stiffness, the water cooling system will have no influential effect on the structure. Finally, it was proven that the effects of cooling systems can have a large influence on the vibration levels and excitation of additional frequencies. Due to this fact, the problem of vibration must be taken into account during the design and integration of new machines. The observation of the additional frequencies is especially important in the $5-80 \mathrm{~Hz}$ spectrum where structures are prone to resonant effects due to the work of other machines in the vicinity. Not measuring the vibration influence on the high precision machines, like accelerators, will effect in the corruption of the experiments data and loss of accuracy. Moreover, for high precision research equipment, which requires the operation of advanced cooling and ventilation it is advisable to use constant monitoring of their influence on the whole system. In this article, it was proven that vibration monitoring is a valid chose for dynamic behaviour evaluation.

\section{References}

1. M. Guinchard, $9^{\text {th }}$ International Particle Accelerator Conference, IPAC18, April 29-May 4, 2018, Vancouver, Canada. S. Koscielniak, Ed. (JACoW, [S.1.]), 2565-2567 (2018)

2. M. Tsypkin, IEEE International Electric Machines and Drives Conference, IEMDC 2011 (2011)

3. Z. K. Peng, F. L. Chu, Mech. Syst. Signal Process (2004)

4. M. Guinchard, $9^{\text {th }}$ International Particle Accelerator Conference, IPAC18, April 29-May 4, 2018, Vancouver, Canada. S. Koscielniak, Ed. (JACoW, [S.1.]), 2568-2570 (2018)

5. C. Cempel, Vib. Phys. Syst. 25, 97-102 (2012)

6. A. Panda, Š. Olejárová, J. Valíček, M. Harničárová, Int. J. Adv. Manuf. Technol. 97, 401-411 (2018)

7. M. Kondo, S. Manabe, T. Takashige, Q. Rep. 105-111 (2016)

8. D. Goyal, B. S. Pabla, CIRP J. Manuf. Sci. Technol. 10, 24-35 (2015)

9. R. B. Randall, Vibration-Based Condition Monitoring: Industrial, Aerospace and Automotive Applications (2010)

10. CERN, "CLIC collaboration, CLIC Conceptual Design Report," (2012)

11. G. Balik, B. Caron, D. Schulte, J. Snuverink, J. Pfingstner, Nucl. Instruments Methods Phys. Res. Sect. A Accel. Spectrometers, Detect. Assoc. Equip. 700, 163-170 (2013)

12. A. Seryi, Particle Accelerator Conference, 2003. PAC (2003)

13. L. Malina, R. Corsini, D. Gamba, T. Persson, P. K. Skowroński, IPAC 2016 Proceedings of the $7^{\text {th }}$ International Particle Accelerator Conference (2016)

14. M. Jashank, D. C. Kala, Int. J. Comput. Appl. 15-18 (2013)

15. Z. Agioutantis, Rock Eng. Rock Mech. Struct. Rock Masses - Proc. EUROCK 2014, ISRM Eur. Reg. Symp. 965-970 (2014)

16. F. Duarte Ramos, F.-X. Nuiry, W. Klempt, Experimental tests on the air cooling of the CLIC vertex detector (2016)

17. A. Moilanen, M. Aicheler, A. Vamvakas, J. Väinölä, S. Doebert, Finite Element Model for Thermal-Structural analysis of CLIC Lab Module type 0\#2 (2017)

18. J. Burjánek, G. Gassner-Stamm, V. Poggi, J. R. Moore, D. Fäh, Geophys. J. Int. (2010) 